\title{
Primeiro registro de machos de Amblyomma rotundatum (Acari: Ixodidae) em jabuti-tinga (Chelonoidis denticulatus) no estado do Amazonas, Amazônia brasileira: relato de caso
}

\author{
[First record of males of Amblyomma rotundatum (Acari: ixodidae) on yellow-footed tortoise (Chelonoidis \\ denticulatus) in the Amazonas state, Brazilian Amazon: case report] \\ S.L. Gianizella ${ }^{1}$, J. Moraes Júnior ${ }^{2}$, C.A.R. Nascimento ${ }^{3}$, T.F. Martins ${ }^{4}$ \\ ${ }^{1}$ Universidade Federal do Amazonas - Manaus, AM \\ ${ }^{2}$ Escola Superior Batista do Amazonas - Manaus, AM \\ ${ }^{3}$ Universidade Federal do Pará - Belém, PA \\ ${ }^{4}$ Universidade de São Paulo - São Paulo, SP
}

\begin{abstract}
RESUMO
O carrapato Amblyomma rotundatum é uma espécie partenogenética, encontrada principalmente em anfíbios e répteis silvestres em todos os biomas brasileiros. No presente relato, registra-se a ocorrência de dois machos dessa espécie de ixodídeo encontrados em um jabuti-tinga (Chelonoidis denticulatus) proveniente do município de Manaus, Amazonas, Brasil.
\end{abstract}

Palavras-chave: carrapatos, Amblyomma rotundatum, machos

\begin{abstract}
The Amblyomma rotundatum tick is a parthenogenetic species, found mainly in wild amphibians and reptiles in all Brazilian biomes. The present report records the occurrence of two males of this ixodid species found on yellow-footed tortoise (Chelonoidis denticulatus) from the municipality of Manaus, Amazonas, Brazil.
\end{abstract}

Keywords: ticks, Amblyomma rotundatum, males

\section{INTRODUÇÃO}

O carrapato Amblyomma rotundatum Koch, 1844, ocorre nas regiões zoogeográficas Neotropical e Neártica, onde parasita principalmente anfíbios e répteis (Guglielmone et al., 2014). No Brasil, essa espécie de carrapato parasita com frequência a herpetofauna brasileira e ocorre em todos os biomas do território nacional, incluindo a Amazônia, Mata Atlântica, Pampa, Cerrado, Pantanal e Caatinga (Aragão, 1936; Woehl Jr., 2002; Brum e Costa, 2003; Szabó et al., 2007; Morais et al., 2010; Horta et al., 2011).

A espécie A. rotundatum se reproduz por partenogênese, e ninfas sempre sofrem ecdise para fêmeas (Aragão, 1936). Por outro lado, existem dois registros de machos no território brasileiro (Labruna et al., 2005; Martins et al., 2014). Curiosamente, um caso de hiperparasitismo nessa espécie de carrapato foi relatado na região Nordeste do país (Labruna et al., 2007). O carrapato $A$. rotundatum é uma espécie próxima a Amblyomma dissimile e Amblyomma goeldii, e todos os estágios já foram descritos (Barros-Battesti et al., 2006).

Junto com o jabuti-piranga (Chelonoidis carbonarius), o jabuti-tinga (Chelonoidis denticulatus) é uma das duas espécies de jabuti que ocorrem no Brasil, sendo encontrado principalmente na região Norte do país, onde é intensamente utilizado como animal de estimação e na alimentação humana. Além do bioma Amazônia, esta espécie de jabuti pode também ser encontrada na Mata Atlântica da região Sudeste do território brasileiro. Ainda no território nacional, o jabuti-tinga pode ser encontrado em menor escala nas regiões Nordeste e Centro-Oeste, habitando o Cerrado e o Pantanal (Vogt et al., 2015).

Recebido em 29 de novembro de 2016

Aceito em 12 de abril de 2017

E-mail: thiagodogo@hotmail.com 


\section{CASUÍSTICA}

No dia 06 de abril de 2016, um jabuti-tinga ( $C$. denticulatus) fêmea adulta, foi atendido em uma clínica veterinária particular, situada no município de Manaus ( $\left.3^{\circ} 6^{\prime} 0^{\prime \prime} \mathrm{S}, 60^{\circ} 1^{\prime} 0^{\prime \prime} \mathrm{W}\right)$, estado do Amazonas (AM), Brasil. O animal, oriundo de uma residência no bairro da Galileia, em Manaus, foi encaminhado ao atendimento clínico com histórico de infestação por carrapatos. Durante a avaliação clínica, constatou-se por inspeção que o réptil apresentava um quadro de parasitismo por carrapatos na região anterior, principalmente nas patas dianteiras. Os exemplares foram removidos com auxílio de pinça e conservados em álcool $70 \%$ para posterior identificação. Os ectoparasitos foram encaminhados para o Laboratório de Zoologia do Departamento de Biologia do Instituto de Ciências Biológicas da Universidade Federal do Amazonas.

Os espécimes foram identificados com auxílio de microscópio estereoscópico com iluminação incidente da marca Olympus, SZX12 - Câmera AmScope MU300 - 5MP. Para a identificação dos ixodídeos, utilizaram-se as chaves taxonômicas para adultos do gênero Amblyomma de Aragão e Fonseca (1961), Guimarães et al. (2001), Barros-Battesti et al. (2006), e, para ninfas desse mesmo gênero, a chave de Martins et al. (2010). Já para os exemplares machos, foi utilizada a descrição de Labruna et al. (2005), segundo a qual os machos apresentaram: formato do corpo oval; sulco marginal ausente; escudo densamente pontuado (especialmente na periferia), ornamentado com manchas pálidoalaranjadas brilhantes presentes ao longo das margens do escudo; base dorsal do capítulo subretangular, com cornuas arredondadas; hipostômio com fórmula dental 3/3; coxas I a IV com dois espinhos curtos e arredondados (Fig. 1). Todos os carrapatos (dois machos, 32 fêmeas e uma ninfa) foram identificados como sendo da espécie A. rotundatum e foram depositados na Coleção Zoológica Paulo Bürhnheim da Universidade Federal do Amazonas (CZPBUFAM), Manaus, AM, Brasil, sob os números de acesso CZPB-IX-000481-000482.

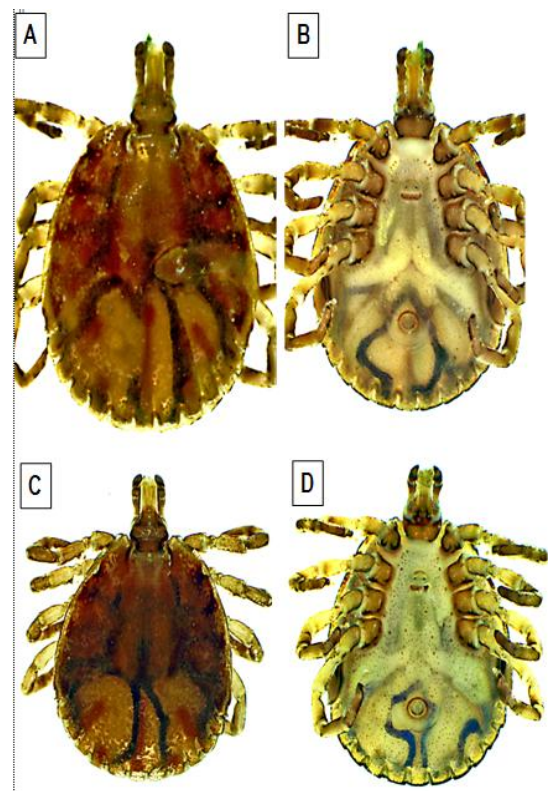

Figura 1. Machos de Amblyomma rotundatum coletados em Chelonoidis denticulatus no município de Manaus, Amazonas, Brasil. Os dois espécimes foram fotografados com microscópio estereoscópico com aumento de 160x. A e C: vista dorsal; B e D vista ventral.

\section{DISCUSSÃO}

Segundo Aragão (1936), o carrapato $A$. rotundatum assim como as espécies $A$. dissimile e A. goeldii são espécies encontradas na região Norte do Brasil, onde predomina o bioma Amazônia. Apesar de Barros-Battesti et al. (2006) relatarem que $A$. rotundatum é uma espécie próxima a $A$. dissimile e $A$. goeldii, no presente estudo foram identificados dois machos de $A$. rotundatum. A principal diferença entre essas três espécies de carrapatos são os espinhos nas coxas I a IV, a ornamentação do escudo e a fórmula dental do hipostômio. O espinho externo da coxa IV é curto e arredondado em $A$. rotundatum, em contraste com o de A. dissimile, que é longo e pontiagudo. Seguindo esse mesmo raciocínio, os machos de $A$. rotundatum apresentam dois espinhos curtos e arredondados nas coxas I a IV, diferenciando-se, assim, de machos de $A$. goeldii, que apresentam dois espinhos apenas na coxa I e um único espinho nas coxas II a IV. A ornamentação em machos de A. rotundatum está presente ao longo das margens do escudo, sendo apenas manchas pálido-alaranjadas brilhantes. Já no caso dos machos de $A$. dissimile, a ornamentação está 
presente por todo o escudo, sendo manchas esbranquiçado-acobreadas, que formam um pseudoescudo de fêmea na porção anterior. Por outro lado, machos de A. goeldii possuem o escudo castanho-escuro, não apresentando ornamentação. Tanto os machos de $A$. rotundatum quanto os machos de $A$. dissimile possuem hipostômio com dentição $3 / 3$, no entanto machos de $A$. goeldii possuem dentição hipostomal 4/4.

No presente estudo, foram identificadas fêmeas e ninfas de $A$. rotundatum em $C$. denticulatus, o que está de acordo com o trabalho recente de Labruna et al. (2010), o quais também relataram fêmeas e ninfas dessa espécie de carrapato nesse mesmo hospedeiro na Amazônia brasileira. Apesar de o carrapato $A$. rotundatum ser encontrado com frequência parasitando animais de sangue frio na região Amazônica brasileira, conforme relatado por Aragão (1936), existem apenas dois relatos de machos dessa espécie de ixodídeo nos limites do território brasileiro (Labruna et al., 2005; Martins et al., 2014). O primeiro relato de Labruna et al. (2005) se refere a um macho coletado na Amazônia em um lagarto de vida livre do gênero Tropidurus sp. no município de Monte Negro, no estado de Rondônia. Curiosamente, o segundo relato de Martins et al. (2014) também ocorreu no mesmo estado, entretanto no município de Porto Velho, sendo um macho coletado em uma serpente jiboia de cativeiro da espécie Boa constrictor. O presente registro de dois machos de $A$. rotundatum em um jabuti domiciliado da espécie C. denticulatus no município de Manaus, estado do Amazonas, corrobora os dois trabalhos prévios realizados no bioma Amazônia. Os poucos registros de machos dessa espécie de carrapato no país evidenciam a necessidade da realização de mais estudos sobre a distribuição geográfica de $A$. rotundatum em diferentes regiões brasileiras e espécies de hospedeiros, principalmente na herpetofauna nacional.

\section{CONCLUSÃO}

Por meio da presente identificação dos ixodídeos, foi possível concluir que machos da espécie do carrapato $A$. rotundatum também estão presentes no estado do Amazonas e foram encontrados parasitando um jabuti-tinga $(C$. denticulatus) na Amazônia brasileira.

\section{AGRADECIMENTOS}

À Fundação de Amparo à Pesquisa do Estado de São Paulo (Fapesp, Projeto 2014/14464-1), Conselho Nacional de Desenvolvimento Científico e Tecnológico (CNPq) e Coordenação de Aperfeiçoamento de Pessoal de Nível Superior (CAPES/PROEX).

\section{REFERÊNCIAS}

ARAGÃO, H. Ixodidas brasileiros e de alguns paizes limitrophes. Mem. Inst. Oswaldo Cruz, v.31, p.759-843, 1936.

ARAGÃO, H.B.; FONSECA, F. Notas de ixodologia VIII: lista e chave para os representantes da fauna ixodológica brasileira. Mem. Inst. Oswaldo Cruz, v.59, p.115-129, 1961.

BARROS-BATTESTI, D.M.; ARZUA, M.; BECHARA, G.H. Carrapatos de importância médico-veterinária da região Neotropical: um guia ilustrado para identificação de espécies. São Paulo: Vox/ICTTD-3/Butantan, 2006. 223p.

BRUM, J.G.W.; COSTA, P.R.P. Confirmação da ocorrência de Amblyomma rotundatum Koch, 1844 (Acari: Ixodidae) no Rio Grande do Sul. Arq. Inst. Biol., v.70, p.105-106, 2003.

GUGLIELMONE, A.A.; ROBBINS, R.G.; APANASKEVICH, D.A. et al. The hard ticks of the world: (Acari: Ixodida: Ixodidae). Dordrecht, Heidelberg, New York: Springer, 2014. 738p.

GUIMARÃES, J.H.; TUCCI, E.C.; BARROSBATTESTI, D.M. Ectoparasitos de importância veterinária. São Paulo: Plêide/Fapesp, 2001. $218 \mathrm{p}$.

HORTA, M.C.; NASCIMENTO, G.F.; MARTINS, T.F. et al. Ticks (Acari: Ixodida) parasitizing free-living wild animals in the Caatinga biome in the State of Pernambuco, northeastern Brazil. Syst. Appl. Acarol., v.16, p.207-211, 2011.

LABRUNA, M.B.; AHID, S.M.M.; SOARES, H.S. et al. Hyperparasitim in Amblyomma rotundatum. J. Parasitol., v.93, p.1531-1532, 2007.

LABRUNA, M.B.; BARBIERI, F.S.; MARTINS, T.F. et al. New tick records in Rondônia, Western Brazilian Amazon. Rev. Bras. Parasitol. Vet., v.19, p.192-194, 2010. 
LABRUNA, M.B.; TERRASSINI, F.A.; CAMARGO, L.M. First report of the male of Amblyomma rotundatum (Acari: Ixodidae) from a field-collected host. J. Med. Entomol., v.42, p.945-947, 2005.

MARTINS, T.F.; ONOFRIO, V.C.; BARROSBATTESTI, D.M. et al. Nymphs of the genus Amblyomma (Acari: Ixodidae) of Brazil: descriptions, redescriptions, and identification key. Ticks Tick Borne Dis., v.1, p.75-99, 2010.

MARTINS, T.F.; VENZAL, J.M.; TERASSINI, F.A. et al. New tick records from the state of Rondônia, western Amazon, Brazil. Exp. Appl. Acarol., v.62, p.121-128, 2014.

MORAIS, D.H.; STRUESSMANN, C.; CARVALHO, V.T. et al. First record of Amblyomma rotundatum Koch, 1844 (Acari: Ixodidae) parasitizing Paleosuchus palpebrosus Cuvier, 1807 (Reptilia: Crocodylidae), in the western border of Pantanal, Mato Grosso do Sul, Brazil. Herpetol. Notes, v.3, p.133, 2010.
SZABÓ, M.P.; OLEGÁRIO, M.M.; SANTOS, A.L. Tick fauna from two locations in the Brazilian savannah. Exp. Appl. Acarol., v.43, p.73-84, 2007.

VOGT, R.C.; FAGUNDES, C.K.; BATAUS, Y.S.L. et al. Avaliação do risco de extinção de Chelonoidis denticulatus (Linnaeus, 1766) no Brasil. Processo de avaliação do risco de extinção da fauna brasileira. ICMBio, 2015. Disponível em: <http://www.icmbio.gov.br/portal/biodiversidade /fauna-brasileira/estado-de-conservacao/7400repteis-chelonoidis-denticulatus-jabutiamarelo.html>. Acessado em: 16 nov. 2016.

WOEHL Jr., G. Infestação de Amblyomma rotundatum (Koch) (Acari, Ixodidae) em sapos Bufo ictericus (Spix) (Amphibia, Bufonidae): novo registro de hospedeiro. Rev. Bras. Zool., v.19, p.329-333, 2002. 